\title{
Development of hybrid reconstruction techniques for TAIGA
}

\section{Blank, ${ }^{a, *}$ M. Tluczykont, ${ }^{a}$ A. Kuotb Awad, ${ }^{a}$ D. Horns ${ }^{a}$ and A. Porelli ${ }^{b}$ on behalf of the TAIGA Collaboration}

(a complete list of authors can be found at the end of the proceedings)

${ }^{a}$ Hamburg University, Institute of Experimental Physics, Luruper Chaussee 149, Hamburg, Germany

${ }^{b}$ Deutsches Elektronen-Synchrotron DESY,

Platanenallee 6, Zeuthen, Germany

E-mail: michael.blank@uni-hamburg.de, martin.tluczykont@uni-hamburg.de, alaa.awad@uni-hamburg.de, dieter.horns@uni-hamburg.de, andrea.porelli@desy.de

The TAIGA-experiment aims to implement a hybrid detection technique of Extensive Air Showers (EAS) at $\mathrm{TeV}$ to $\mathrm{PeV}$ energies, combining the wide angle Cherenkov timing array HiSCORE with Imaging Air Cherenkov Telescopes (IACTs). The detector currently consists of 89 HiSCORE stations and two IACTs, distributed over an area of about $1 \mathrm{~km}^{2}$. Our goal is to introduce a new reconstruction technique, combining the good angular and shower core resolution of HiSCORE with the gamma-hadron separation power of the imaging telescopes. With the second IACT in operation, three different event types can be explored: IACT stereo, full hybrid (IACT stereo + stations) and mono hybrid (IACT mono + HiSCORE), the latter being the operational goal of TAIGA. The status of the development of the full hybrid reconstruction and its verification using real data and simulation are presented.

$37^{\text {th }}$ International Cosmic Ray Conference (ICRC 2021)

July 12th - 23rd, 2021

Online - Berlin, Germany

\footnotetext{
${ }^{*}$ Presenter
} 


\section{Introduction}

Gamma Rays at energies above a few tens of $\mathrm{TeV}$ are a promising area for numerous topics ranging from better understanding the physics of the objects emitting them to dark matter searches to EBL constraints and more. With the latest findings of LHAASO [1], it became evident that observations at these highest energies pose some prospect. However, experiments aiming to achieve useful sensitivities require effective areas of a few $\mathrm{km}^{2}$. The Tunka Advanced Instrument for cosmic ray and Gamma ray Astronomy (TAIGA) aims to establish the combination of Imaging Air Cherenkov Telescopes (IACTs) with the light front sampling optical timing stations of HiSCORE to cost-effectively cover $1 \mathrm{~km}^{2}$ in a first and $5 \mathrm{~km}^{2}$ in a second stage[2,3].

With 88 stations in operation, the first IACT reasonably well understood and the second IACT installed in february of 2020, the new event type of Stereo IACT + HiSCORE has become available for exploration.

In this work, a first look at the Core position estimates for Stereo IACTs and the HiSCORE array is given and compared for a real data run. Furthermore, the expected reconstruction accuracy is investigated on a set of simulated Gammas.

\section{The TAIGA Project}

The Tunka Advanced Instrument for cosmic ray and Gamma ray Astronomy (TAIGA) focusses on establishing the hybrid detection techniques at $\mathrm{TeV}$ to $\mathrm{PeV}$ energies. It was conceived and initiated about 10 years ago. The mainly russian-german project is located on the site of the Tunka133 experiment [4] and aims to combine several subprojects. The main projects are the imaging air Cherenkov telescopes (IACTs) of the $4 \mathrm{~m}$ class, the light front sampling HiSCORE array [5], and underground muon detectors. For more details of the project, see the overview talk of this conference [3]. One of the goals of TAIGA is to establish the hybrid method, which aims to combine the gamma/hadron separation power of IACT images with the core position and origin estimation of the HiSCORE array. The array is expected to reach a size of 120 optical stations spaced by $106 \mathrm{~m}$ and three IACTs spaced by $\approx 300 \mathrm{~m}$, covering $1 \mathrm{~km}^{2}$ by the end of 2021 .

\section{The Hybrid Cherenkov Approach}

The traditional stereoscopic reconstruction approach of IACT arrays requires that one event triggers at least two telescopes, forcing a close spacing of the telescopes of about $50 \mathrm{~m}$ to about $200 \mathrm{~m}$. For the hybrid approach, where the core location is derived from the HiSCORE stations, the distance between IACTs spread over the array can be increased to about $600 \mathrm{~m}$. This allows to cover large areas at lower cost. One example hybrid event is shown in Fig. 2b

One of the advantages of the hybrid approach is the independence of the core determination from the distance to the telescope. Where stereoscopic systems suffer from bad stereo-angles at large core distances, TAIGA uses the HiSCORE array for the reconstruction of the core impact position.

This combination of different instruments enables new reconstruction possibilities. For example, the major axis could be fixed by the station core estimate and not by the image moments. One new approach which was already studied is the Hybrid scaled width. 


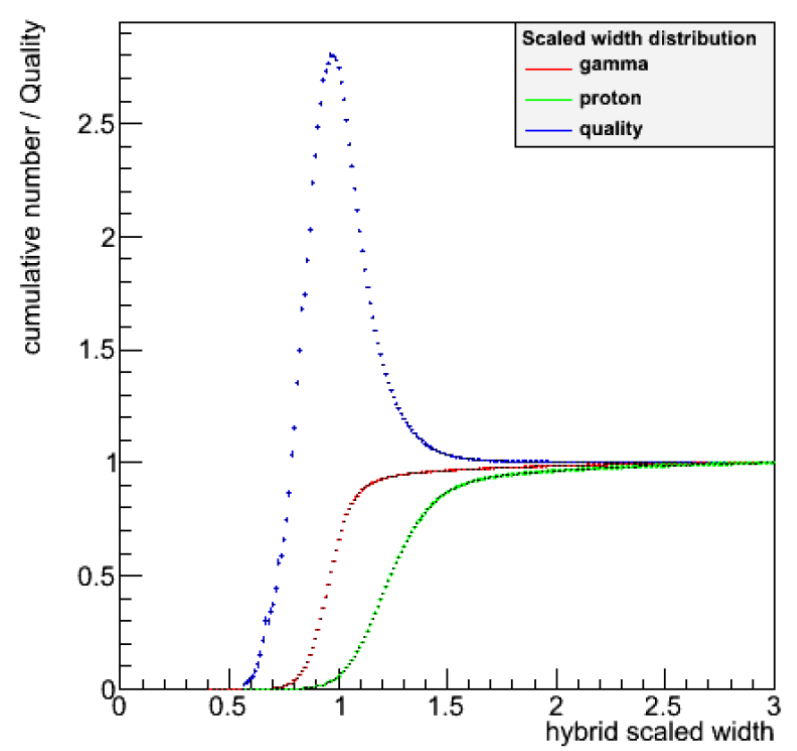

Figure 1: Quality factor against the hybrid scaled width cut value of a TAIGA-IACT-like camera as obtained by [6]. This plot was obtained by confining the center of gravity of the light distribution in the camera to $<4^{\circ}$, the simulated energy to $>20 \mathrm{TeV}$ and the core distance to $<300 \mathrm{~m}$.

In classical stereoscopy, one widely used parameter for gamma / hadron separation is the Scaled Width (SCW), which is the width measured by the telescope divided by the width expectation for that image size for an event at the reconstructed core position. For TAIGA, the same approach, but using the array reconstructed core estimate can be used. This approach was termed Hybrid Scaled Width (HSCW) and was investigated on simulations in [6] and found to improve the quality factor from $\mathrm{q}=2$ using only width to $\mathrm{q}=2.8$. Fig. 1 shows the obtained quality factors for different cut choices of the HSCW.

\section{Stereo and HiSCORE events}

With the second IACT in operation, events triggering both telescopes and the array have become available for exploration. This event class is well suited for verification and calibration purposes between the IACTs and HiSCORE. One example of possible calibration and performance checks is to compare the reconstructed core positions of the HiSCORE array with that obtained by the stereoscopic approach.

In total 150 minutes of simultaneous IACT and HiSCORE data from the observation night of 2020-02-20 with a zenith angle from $30^{\circ}$ to $43^{\circ}$ were used for this analysis. The air shower simulations are conducted with CORSIKA v7.64[7] with the IACT extension. For the IACT simulation, an adapted version of sim_telarray[8] and for the HiSCORE array, sim_score was written based on the sim_skeleton example program of the sim_telarray project. The further reconstruction steps are performed in python with the same tools as used for real data.

For the IACTs, an image cleaning is performed, then classical Hillas parameters are calculated. The basic approach for the calculation of the stereoscopic impact point is then done as follows: 


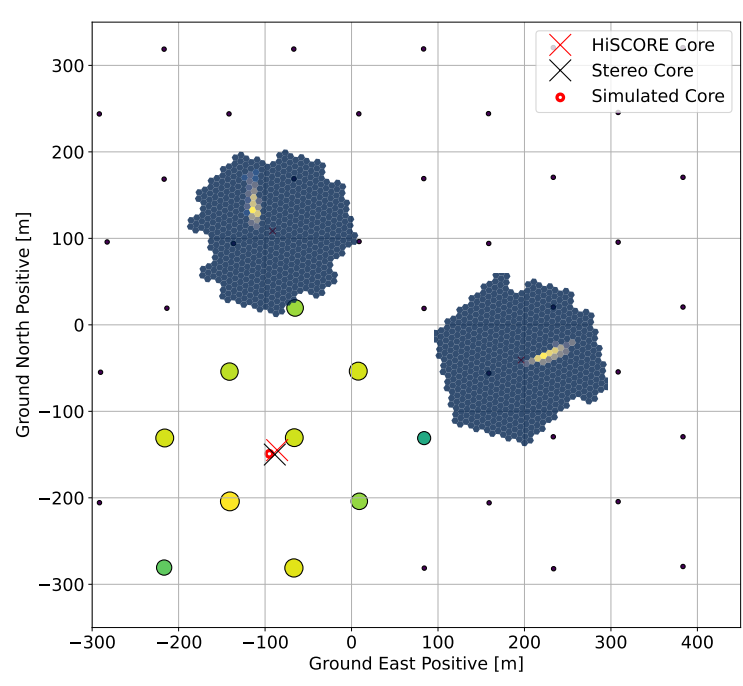

(a) Golden event

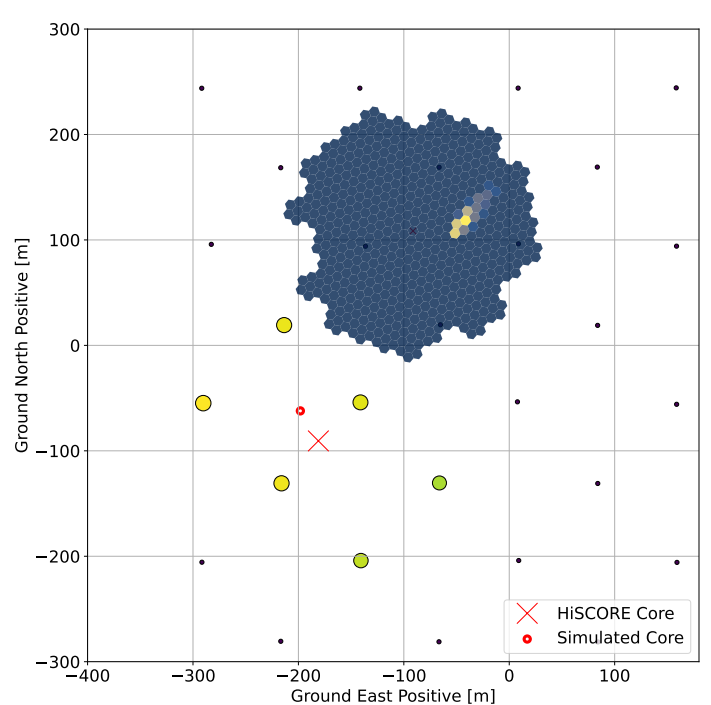

(b) Operational goal

Figure 2: Two example events of array with iacts. (a) shows an example event of one IACT and array. These event types are the operational goal of TAIGA. (b) shows an event where two IACTs and the array trigger. These types of event are called the golden events.

Calculate the corresponding altitude and azimuth for the Center of Gravity of the light distribution. Calculate the altitude and azimuth for a position one unit length along the reconstructed major axis. Determine the unit vectors corresponding to these two pointings at the telescope positions and calculate the plane through the shower axis and the telescope position. Finally, both planes are intersected at the observation height. An example of an event lending itself very well for the stereoscopic reconstruction is shown in Fig. 2a. Fig. 3a shows the distribution of impact points obtained for the data sample. When compared to the reconstructed simulation sample shown in Fig. 3b, a similar structure can be seen. Both distributions show the void areas close to the connection line of the telescopes. Along these, the approach used here is not suitable. The difference in the overall shape, where the simulated data looks more elongated than the simulation can be explained by the different azimuth angles and zenith distances. The simulation was calculated at a zenith distance and azimuth of $31^{\circ}$ and $180^{\circ}$.

The core positions measured by the array are determined via the Center of Gravity of the measured charge per station $(\mathrm{CoG}$ or $\mathrm{CoQ})$. The measured charge is the integral of the pulse sampled by the station. The CoG method can be used with as few as 2 stations and is quite robust against fluctuations. An alternative with better core resolution (with enough stations triggering) would be to use a fit to the lateral light density function, but this is not done here because of the robustness, as most of the events in this sample trigger only very few stations. In this first approach, the data is taken without any calibration, highlighting the robustness of this method.

For a common sample of events which trigger both IACTs and HiSCORE, Fig. 4 shows the reconstructed core positions for both methods. For the stereo reconstruction in Fig. 4a, the events far outside of the array boundary are unlikely to be correctly reconstructed because the events 


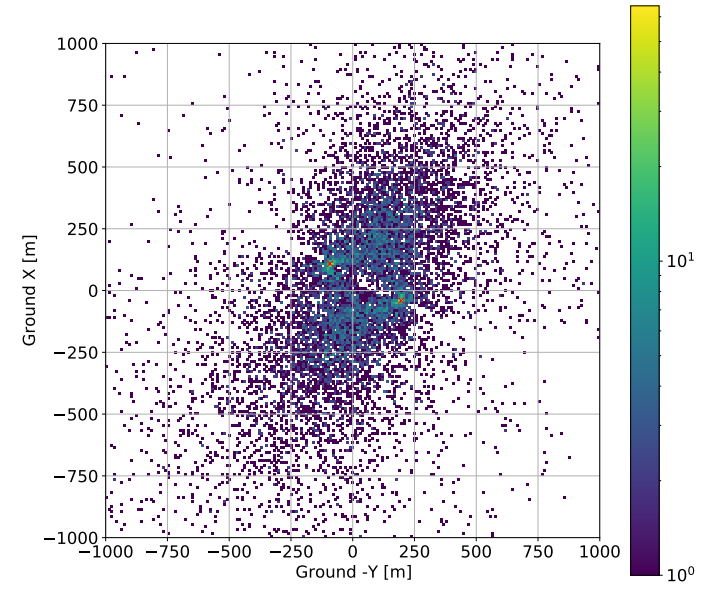

(a) Data

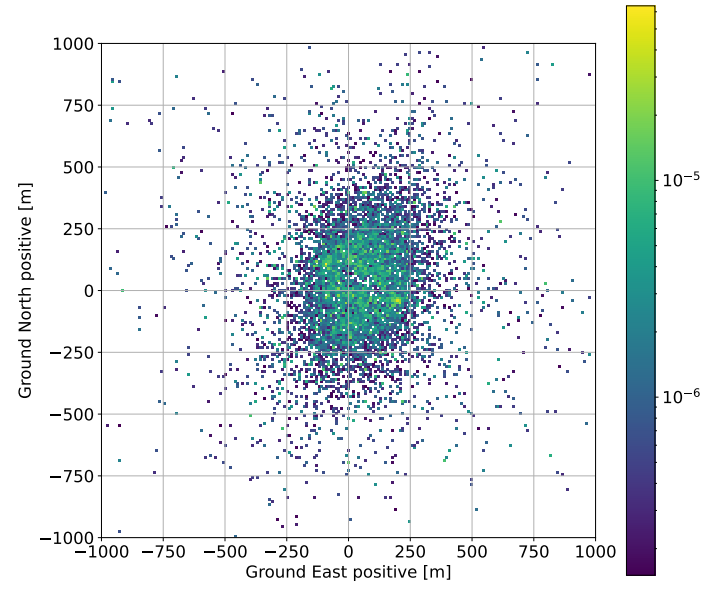

(b) Simulation

Figure 3: Reconstructed impact locations of stereo real data events (a) and simulated Hadrons (b). The simulation sample was composed via weighting after the Gonato model with the following particle species: proton, helium, nitrogen and iron. The difference of the shapes can be at least partly explained by the different azimuth and zenith angles. While (a) was observed at a range of azimuth and zenith angles with a mean azimuth of $210^{\circ}$ and a mean zenith distance of $0^{\circ}$, (b) was created using a single orientation with an azimuth of $180^{\circ}$ and a zenith distance of $31^{\circ}$.

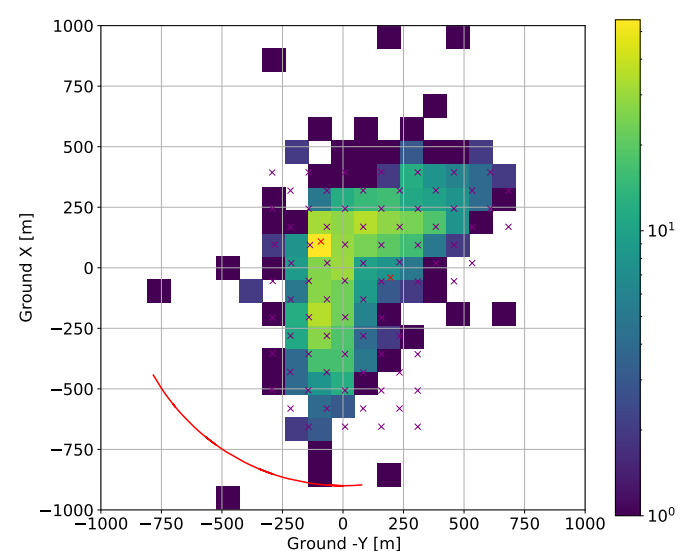

(a) Stereo reconstruction

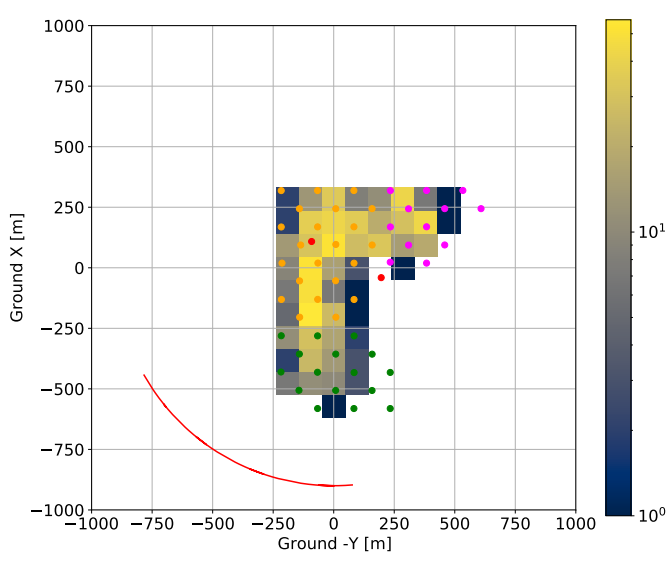

(b) HiSCORE Center of Gravity

Figure 4: Reconstructed impact locations of coincident real data events. (a) shows the impact estimation obtained via a stereoscopic approach. (b) depicts the impact estimations for the same events as obtained by HiSCORE via the center of gravity of the charge calculation. The red markers show the position of the two IACTs. The violet crosses in (a) show the location of the active stations, while the markers in (b) show the remaining stations after exclusion of the border stations. The red circles depict the telescope locations, while each of the other colors corresponds to a different HiSCORE cluster. The red line is a visualization of the IACTs azimuth angles during the observation. 


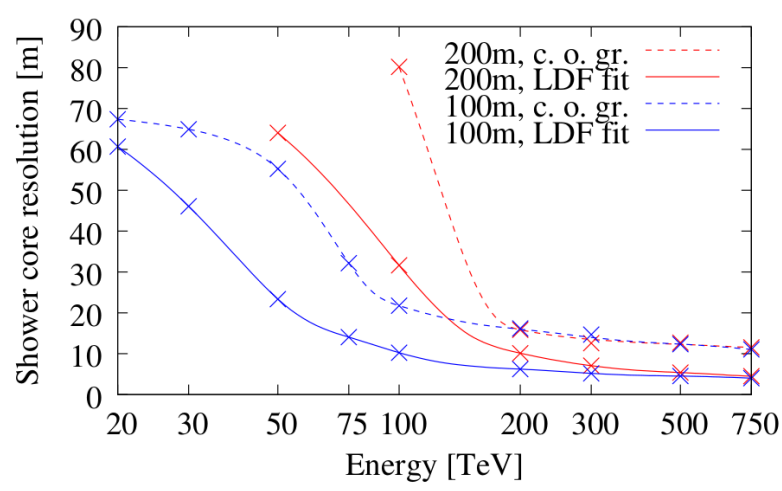

Figure 5: Expected resolutions for regular arrays with $100 \mathrm{~m}$ and $200 \mathrm{~m}$ spacing at $0 \mathrm{~m}$ above sea level and zenith distances of $0 \mathrm{deg}$ to $20 \mathrm{deg}$ as obtained in [9]. For the energy range of $20 \mathrm{TeV}$ to $50 \mathrm{TeV}$, the expected resolution for the real HiSCORE array with a spacing of about $106 \mathrm{~m}$ using the center of gravity method would be $\approx 65 \mathrm{~m}$.

which trigger the boundary stations are excluded and the light pool at the core location is likely to be contained within the array. The large number of events reconstructed to the location of the first IACT is likely a result of the bad stereo angles when the core location is along the connection line but closer to IACT 1. A pile up at the IACT 2 position is not visible because most of the events here are cut away by the exclusion of the events with border stations, leaving the location of IACT 2 well outside of the remaining array. The irregularities seen in Fig. $4 \mathrm{~b}$ might be a result of taking the charge of the stations - in this first approach - without calibration. To study the the expected reconstruction accuracy, we use a set of Gamma simulations.

\subsection{Simulated Core Resolution}

Fig. 6a shows the histogram of the distance of the stereoscopically reconstructed core position to the one reconstructed via the array for Gamma simulations between $\approx 20 \mathrm{TeV}$ and $50 \mathrm{TeV}$. The corresponding cumulative distribution is shown in green in Fig. 6b. The expected resolution for the stereo core position to array $\mathrm{CoG}$ core position is $\approx 70 \mathrm{~m}$. The orange curve corresponds to the distance of the true (simulated) core position to the station CoG reconstructed one and the blue curve is the corresponding distribution for the used stereo method. For this sample, the CoG resolution is $\approx 40 \mathrm{~m}$ and it is even slightly better than the Stereo reconstructed one. Also, the tail of core positions misreconstructed by $>200 \mathrm{~m}$ core is not present for the array reconstruction. This is very promising for possible reconstruction methods for the one IACT + HiSCORE event class, which is the operational goal of TAIGA.

The resolution of $\approx 40 \mathrm{~m}$ at $\approx 20 \mathrm{TeV}$ to $50 \mathrm{TeV}$ for the $\mathrm{CoG}$ method with the array spacing of $\approx 106 \mathrm{~m}$ is better than the expected value of $\approx 65 \mathrm{~m}$ for an array with a spacing of $100 \mathrm{~m}$ (see Fig. 5) obtained in the first simulations in [9].

\section{Summary and Outlook}

With the second IACT in operation, the Stereo + HiSCORE events have become available. The coincident events are seen in data and $\mathrm{MC}$ and the obtained core resolution of $\approx 40 \mathrm{~m}$ at $\approx 20 \mathrm{TeV}$ 


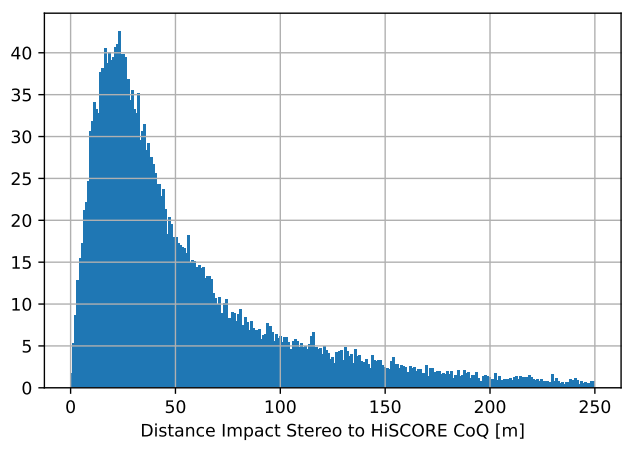

(a) Histogram

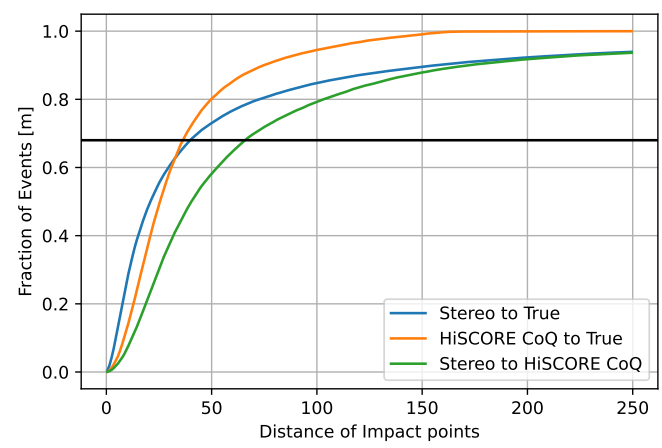

(b) Cumulative distance

Figure 6: (a) shows the histogram of the stereo reconstructed core position to the core position reconstructed via the HiSCORE array with the center of gravity method. The green curve in (b) corresponds to the histogram in (a). The orange curve corresponds to the distance of the true (simulated) core position to the one reconstructed with the array and the blue curve is the corresponding distribution for the stereoscopically reconstructed events.

to $50 \mathrm{TeV}$ is better than first expected. These results are a good sanity check and a promising step on the path to the reconstruction of Single IACT + HiSCORE events with techniques such as the hybrid scaled width method.

\section{Acknowledgments}

The work was performed at the UNU "Astrophysical Complex of MSU-ISU". The work is supported by Russian Foundation for Basic Research (grants 19-52-44002, 19-32-60003), the Russian Science Foundation (grant 19-72-20067 (Section D,E), the Russian Federation Ministry of Science and High Education (projects FZZE-2020-0017, FZZE-2020-0024), by the Deutsche Forschungsgemeinschaft (DFG, TL 51/6-1) and by the Helmholtz Association 13 (HRJRG-303) and by European Union's Horizon 2020 programme (No.653477). D.H., M.T, A.K.A. and M.B. acknowledge support by the Deutsche Forschungsgemeinschaft (DFG, German Research Foundation) under Germany's Excellence Strategy — EXC 2121 "Quantum Universe" - 390833306.

\section{References}

[1] Z. Cao, F.A. Aharonian, Q. An, L.X. Axikegu, Bai, Y.X. Bai, Y.W. Bao et al., Ultrahigh-energy photons up to 1.4 petaelectronvolts from 12 gamma-ray Galactic sources, Nature 594 (2021) 33.

[2] N. Budnev, I. Astapov, P. Bezyazeekov, A. Bonvech, A. Borodin, M. Brueckner et al., Status of the taiga experiment: From cosmic-ray physics to gamma astronomy in tunka valley., Phys. Atom. Nuclei 83 (2020) 905-915.

[3] N. Budnev and (TAIGA collaboration), The taiga - an advanced hybrid detector complex for astroparticle physics, cosmic ray physics and gamma-ray astronomy, this conference (2021) . 
[4] S.F. Berezhnev, D. Besson, N.M. Budnev, A. Chiavassa, O.A. Chvalaev, O.A. Gress et al., The Tunka-133 EAS Cherenkov light array: Status of 2011, NIMA 692 (2012) 98.

[5] M. Tluczykont, D. Hampf, D. Horns, D. Spitschan, L. Kuzmichev, V. Prosin et al., The HiSCORE concept for gamma-ray and cosmic-ray astrophysics beyond 10TeV, Astroparticle Physics 56 (2014) 42.

[6] M. Kunnas, "Studies of the performance of an iact system for the taiga array." https://ediss.sub.uni-hamburg.de/handle/ediss/7582.

[7] D. Heck, J. Knapp, J.N. Capdevielle, G. Schatz and T. Thouw, CORSIKA: a Monte Carlo code to simulate extensive air showers. (1998).

[8] K. Bernlöhr, Simulation of imaging atmospheric Cherenkov telescopes with CORSIKA and sim_telarray, Astroparticle Physics 30 (2008) 149 [0808.2253].

[9] D. Hampf, M. Tluczykont and D. Horns, Event reconstruction with the proposed large area Cherenkov air shower detector SCORE, arXiv e-prints (2009) arXiv:0909.0663 [0909. 0663].

\section{Full Authors List: TAIGA Collaboration}

M. Blank ${ }^{10}$ M. Tluczykont ${ }^{10}$ A. K. Awad ${ }^{10}$ D. Horns $^{10}$ A. Porelli ${ }^{5}$ I. I. Astapov ${ }^{2}$ P. A. Bezyazeekov ${ }^{3}$ E. A. Bonvech ${ }^{1}$ A. N. Borodin $^{4}$ A. V. Bulan ${ }^{1}$ M. Brueckner ${ }^{5}$ N. M. Budnev ${ }^{3}$ A. Chiavassa ${ }^{6}$ D. V. Chernov ${ }^{1}$ A. N. Dyachok ${ }^{3}$ A. R. Gafarov ${ }^{3}$ A. Yu. Garmash ${ }^{7,8}$ V. M. Grebenyuk ${ }^{4,9}$ O. A. Gress ${ }^{3}$ E. Gress ${ }^{3}$ T. I. Gress ${ }^{3}$ O. G. Grishin ${ }^{3}$ A. A. Grinyuk ${ }^{4}$ N. N. Kalmykov ${ }^{1}$ V. V. Kindin ${ }^{2}$ S. N. Kiryuhin ${ }^{3}$ R. P. Kokoulin ${ }^{2}$ K. G. Kompaniets ${ }^{2}$ E. E. Korosteleva ${ }^{1}$ V. A. Kozhin ${ }^{1}$ E. A. Kravchenko ${ }^{7,8}$ A. P. Kryukov ${ }^{1}$ L. A. Kuzmichev ${ }^{1}$ A. A. Lagutin ${ }^{11}$ M. Lavrova ${ }^{4}$ B. K. Lubsandorzhiev ${ }^{12}$ N. B. Lubsandorzhiev ${ }^{1}$ A. D. Lukanov ${ }^{12}$ D. S. Lukyantsev ${ }^{3}$ R. R. Mirgazov ${ }^{3}$ R. Mirzoyan $^{13,3}$ R. D. Monkhoev ${ }^{3}$ E. A. Osipova ${ }^{1}$ A. L. Pakhorukov ${ }^{3}$ A. Pan ${ }^{4}$ L. V. Pankov ${ }^{3}$ A. D. Panov ${ }^{1}$ A. A. Petrukhin ${ }^{2}$ D. A. Podgrudkov $^{1}$ V. A. Poleschuk ${ }^{3}$ M. Popesku ${ }^{14}$ E. G. Popova ${ }^{1}$ E. B. Postnikov ${ }^{1}$ V. V. Prosin ${ }^{1}$ V. S. Ptuskin ${ }^{15}$ A. A. Pushnin ${ }^{3}$ R. I. Raikin ${ }^{11}$ A. Y. Razumov ${ }^{1}$ G. I. Rubtsov ${ }^{12}$ E. V. Ryabov ${ }^{3}$ Y. I. $\operatorname{Sagan}^{4,9}$ V. S. Samoliga ${ }^{3}$ A. A. Silaev ${ }^{1}$ A. A. Silaev(junior) ${ }^{1}$ A. Yu. Sidorenkov ${ }^{12}$ A. V. Skurikhin ${ }^{1}$ M. Slunecka ${ }^{4}$ A. V. Sokolov ${ }^{7,8}$ L. G. Sveshnikova ${ }^{1}$ V. A. Tabolenko ${ }^{3}$ B. A. Tarashansky ${ }^{3}$ L. G. Tkachev ${ }^{4,9}$ R. $\operatorname{Togoo}^{16}$ N. Ushakov ${ }^{12}$ A. Vaidyanathan ${ }^{7}$ P. A. Volchugov ${ }^{1}$ N. V. Volkov ${ }^{11}$ D. Voronin ${ }^{12}$ R. Wischnewski ${ }^{5}$ A. V. Zagorodnikov ${ }^{3}$ D. P. Zhurov ${ }^{3,17}$ I. I. Yashin ${ }^{2}$ Skobeltsyn Institute of Nuclear Physics, Moscow State University, Moscow, 119991 Russia ${ }^{2}$ National Research Nuclear University MEPhI, Moscow, 115409 Russia ${ }^{3}$ Research Institute of Applied Physics, Irkutsk State University, Irkutsk, 664003 Russia ${ }^{4}$ Joint Institute for Nuclear Research, Dubna, Moscow oblast, 141980 Russia ${ }^{5}$ Deutsches Elektronen-Synchrotron DESY, Zeuthen, 15738 Germany ${ }^{6}$ Physics Department of the University of Torino and Istituto Nazionale di Fisica Nucleare, Torino, 10125 Italy ${ }^{7}$ Novosibirsk State University, Novosibirsk, 630090 Russia ${ }^{8}$ Budker Institute of Nuclear Physics, Siberian Branch, Russian Academy of Sciences, Novosibirsk, 630090 Russia ${ }^{9}$ Dubna University, Dubna, Moscow oblast, 141980 Russia ${ }^{10}$ Institut für Experimentalphysik, Universität Hamburg, Hamburg, D-22 761 Germany ${ }^{11}$ Altai State University, Barnaul, Altai krai, 656049 Russia ${ }^{12}$ Institute for Nuclear Research, Russian Academy of Sciences, Moscow, 117312 Russia ${ }^{13}$ Max Planck Institute for Physics, Munich, 80805 Germany ${ }^{14}$ Space Science Institute, Magurele, 077125 Romania ${ }^{15}$ Pushkov Institute of Terrestrial Magnetism, Ionosphere and Radio Wave Propagation, Russian Academy of Sciences, Troitsk, Moscow, 142190 Russia ${ }^{16}$ Institute of Physics and Technology Mongolian Academy of Sciences, Ulaanbaatar, Mongolia ${ }^{17}$ Irkutsk National Research Technical University, Irkutsk, Russia 